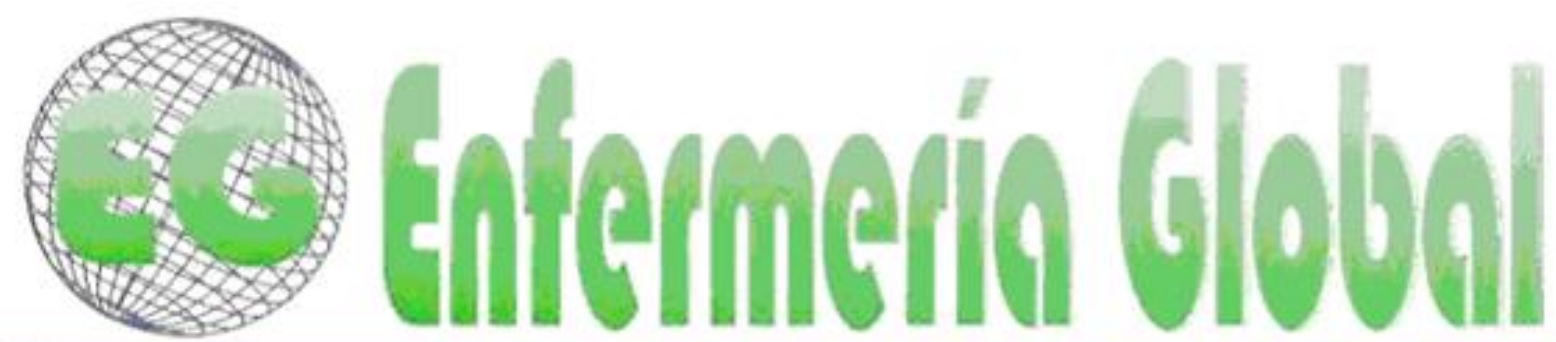

ISSN 1695-6141

Revista electrónica trimestral de Enfermería

$N^{\circ} 44$

Octubre 2016

www.um.es/egloball

\title{
CLÍNICA
}

\section{Vivencias de las jóvenes diagnosticadas de escoliosis idiopática}

Experiences of young diagnosed with idiopathic scoliosis

\section{${ }^{*}$ Carrasco, $\mathrm{M}^{\mathrm{a}}$ Isabel Bonilla ${ }^{* *}$ Ruiz, $\mathrm{M}^{\mathrm{a}}$ Carmen Solano}

\author{
*Enfermera Asistencial Hospital Traumatología y Rehabilitación Vall d’Hebron. Profesora Asociada, \\ Departamento de Enfermería, Universidad Autónoma de Barcelona. E-mail: davibel3@hotmail.com \\ **Profesora Titular de Enfermería. Facultad de Ciencias de la Salud. Universidad de Alicante. España.
}

Palabras clave: escoliosis; adolescente; imagen corporal; autopercepción; mociones.

Keywords: scoliosis; adolescent body image; self-perception; emotions

\section{RESUMEN}

Justificación: Durante la adolescencia y la juventud acontecen cambios psicológicos y fisiológicos que forman parte del desarrollo normal de la persona. La irrupción de la escoliosis en esta edad influye en la vivencia de la autopercepción corporal.

Objetivo: Conocer cómo una deformidad corporal influye en la estabilidad emocional de las jóvenes diagnosticadas de escoliosis.

Diseño: Metodología cualitativa fenomenológicas situada en la hermenéutica.

Método: Se utilizó una entrevista semiestructurada y dirigida a mujeres jóvenes con escoliosis idiopática ingresadas en la unidad de raquis del Hospital Vall d’Hebron en Barcelona.

Resultados: Los principales resultados señalan un compromiso emocional que afecta a las actividades de la vida diaria de las jóvenes, manifestándose, en algunas ocasiones, con sentimientos de vergüenza, tristeza, preocupación, miedo, amargura, complejo y rabia. La experiencia se vive como un duelo ante la pérdida de expectativas futuras.

Conclusiones: Los sentimientos y las emociones vividas y referidas por estas pacientes comprometen su estabilidad emocional.

\section{ABSTRACT}

Justification: During adolescence and youth psychological and physiological changes occur that are part of the normal development of the individual. The emergence of scoliosis in this age influences the perception of body experience. 
Background: Knowing how a bodily deformity affects the emotional stability of young people diagnosed with scoliosis.

Design: Qualitative research with phenomenological methodology at the hermeneutics.

Method: A semi-structured interview was used and aimed at young women with idiopathic scoliosis entered in the spine unit at the Hospital Vall d'Hebron in Barcelona.

Results: The main results indicate an emotional commitment that affects the activities of daily life for young people, demonstrating, sometimes with feelings of shame, sadness, worry, fear, bitterness, and rage complex. The experience is lived as a duel to the loss of future expectations.

Conclusions: Feelings and emotions experienced and reported by these patients commit their emotional stability.

\section{INTRODUCCIÓN}

Cuando se habla de imagen corporal en relación con la etapa de la adolescencia en el ser humano, el aspecto más destacable es la espectacularidad de los cambios corporales. Esta evidencia corporal exterior tiene siempre un acompañamiento intrapsíquico. El descubrimiento del cuerpo se encarna para los adolescentes en experiencias inolvidables que marcarán el resto de su vida ${ }^{(1)}$. La irrupción de una deformidad física durante este período de crecimiento y de construcción de la identidad supondrá la vivencia de unas experiencias que van a influenciar la autoestima y la salud mental. La visión de la imperfección en el cuerpo humano produce ambivalencia en el espectador, entre rechazo al que la padece y humanidad hacia su defecto ${ }^{(2)}$.

Los estudios que mayoritariamente se utilizan para la valoración de las diferentes dimensiones que conforman la escoliosis son de tipo cuantitativo, en ellos se han utilizado varios métodos para medir la magnitud de la deformidad. Uno de los enfoques consiste en solicitar la impresión personal del paciente respecto a su deformidad mediante cuestionarios ${ }^{(3)}$. El objetivo es intentar medir cómo ven su cuerpo en relación con la curvatura de su espalda ${ }^{(4)}$. Las dimensiones más importantes utilizadas en estos cuestionarios son el dolor, la imagen corporal, la movilidad, los trastornos del sueño y una combinación de factores que hacen referencia a la funcionalidad en general ${ }^{(5,6)}$, poniendo de manifiesto la reducción de la calidad de vida en los pacientes afectados de escoliosis y relacionando de forma específica la depresión, el dolor de espalda, la capacidad física limitada y una negativa autopercepción corporal con la deformidad ${ }^{(7)}$.

Los cuestionarios específicos, en general, intentan medir y cuantificar la percepción de la deformidad corporal que tienen las mujeres afectas de escoliosis idiopática. Para algunos investigadores la puntuación de la propia imagen tiene el coeficiente de correlación más alta con la corrección de la curva, pero no pueden afirmar que las curvas más grandes están menos correlacionadas o que los pacientes son más sensibles en su forma corporal debido a su mayor deformidad. Sanders et al. no ha encontrado correlación entre los puntajes de imagen corporal del SRS y la magnitud de la curva en el postoperatorio ${ }^{(8)}$. Los resultados de los estudios de Parent et al. permiten afirmar que cuestionarios específicos como el SRS-22 no pueden detectar diferencias entre personas con diferencias en la severidad de la curva o entre aquellos sometidos a diferentes tratamientos conservadores ${ }^{(9)}$. En este sentido, 
existen estudios que sugieren también que la relación entre la deformidad y la percepción de la imagen corporal, el dolor y la función es insignificante ${ }^{(3)}$.

Se han encontrado muy pocos estudios de metodología cualitativa que concluyan cómo se ven a sí mismas estas jóvenes o cómo viven toda la sintomatología asociada a esta deformidad antes, durante o después de un tratamiento definitivo. Un ejemplo de ello es el estudio de Agata et al., que intenta relacionar la autopercepción corporal con el estrés emocional que presentan los pacientes escolióticos. En este estudio se analizan y valoran los dibujos relacionados con la figura humana escoliótica, y se llega a la conclusión de que la representación del sombreado de hombros puede asociarse al estrés emocional de los pacientes. En el caso de la omisión de los rasgos faciales o bien la omisión completa de características de todo el cuerpo de la figura humana que se dibuja, se concluye que tienen un valor de evasivas y superficialidad, y podría también expresar una posible tendencia al aislamiento social de estos pacientes $^{(10)}$.

El objetivo de este estudio es conocer cómo una deformidad corporal influye en la estabilidad emocional de las jóvenes diagnosticadas de escoliosis.

\section{MATERIAL Y MÉTODO}

Se lleva a término un estudio de tipo cualitativo fenomenológico de orientación hermenéutica con el objetivo de profundizar en la experiencia humana ${ }^{(11)}$ de jóvenes afectadas por EIA.

La población objeto de estudio son todas aquellas jóvenes de edades comprendidas entre 15 y 24 años, diagnosticadas de escoliosis idiopática, que ingresan en la unidad asistencial de Cirugía de Raquis del Hospital de Traumatologia y Rehabilitación Vall d’Hebron para ser sometidas a una intervención quirúrgica como medida de tratamiento a su deformidad.

El estudio se lleva a término durante el período de junio de 2012 a septiembre de 2014. El tamaño de la muestra es de 16 jóvenes que cumplen los criterios de inclusión. La muestra estuvo condicionada por el criterio de saturación de los datos obtenidos tras un análisis previo ${ }^{(12)}$. Según este criterio teórico de saturación, el tamaño de la muestra ha sido incrementado hasta que se ha logrado el nivel de saturación ${ }^{(13)}$. Se utiliza como captador a un total de dos enfermeras referentes de la unidad de raquis a las que se les proporcionan las características de los sujetos a estudiar y los criterios de inclusión y exclusión.

El criterio de selección muestral no tiene como propósito representar una población a partir de la cual se puedan generalizar resultados. La muestra siempre es intencional y su selección ha sido determinada por la amplitud, variedad e integración de las diversas realidades que convergen en el objeto estudiado ${ }^{(14)}$. Se trata de un muestreo opinático donde se han seguido criterios estratégicos personales en la selección de las participantes, como por ejemplo que estuviesen hospitalizadas en el momento de la entrevista.

Criterios de inclusión: Todas la jóvenes diagnosticadas de EIA, de sexo femenino, con una edad comprendida entre 15 y 24 años, ingresadas en la unidad de raquis del Hospital de Traumatología y Rehabilitación de Vall d’Hebron para un tratamiento 
quirúrgico de su deformidad. También las jóvenes que acuden a consultas externas del mismo centro para control de la evolución de su curvatura escoliótica. Se decide utilizar sólo pacientes de sexo femenino debido a que la prevalencia de la escoliosis idiopática adolescente (EIA) se aproxima al $2 \%$ de la población, con una frecuencia cinco veces mayor en niñas que en niños ${ }^{(15)}$.

Criterios de exclusión: Quedarán excluidas las pacientes diagnosticadas de EIA con edad inferior a los 15 años o aquellas que sobrepasen la edad de 24 años, también aquellas pacientes que llevan asociada a su escoliosis otro tipo de patología, como por ejemplo una parálisis cerebral o una deficiencia psíquica. Se excluirán también las pacientes con intervenciones por infecciones recurrentes o por rotura del material de osteosíntesis.

Aspectos éticos: Se solicitó la aprobación del estudio por el comité ético de investigación clínica y comisión de proyectos de investigación del Hospital Universitario Vall d’Hebron, siendo aprobado en la sesión ordinaria nำ185 de marzo del 2011.

Previo a la recogida de datos, se explicó a las participantes el objetivo del estudio y se solicitó su participación de forma voluntaria. Se garantizó la confidencialidad de la información recogida, de acuerdo con las recomendaciones del comité ético de investigación del centro, suministrando a cada paciente y a su tutor referente un modelo de consentimiento informado y una hoja informativa sobre estudios sin procedimientos invasivos, ambos documentos recomendados por dicho comité. Se ha facilitado también información sobre el trato de la información recogida y sobre la destrucción de las grabaciones auditivas realizadas durante las entrevistas.

Las entrevistas grabadas se transcribieron literalmente para su posterior análisis, utilizándose el soporte técnico de un programa informático, Atlas Ti 6.2., y estableciéndose categorías y unidades de sentido para facilitar su comprensión y manejo a nivel práctico y teniendo en cuenta los objetivos del estudio inicial ${ }^{(16)}$. También se marcó a todas las participantes con una etiqueta identificativa de " $A$ " $\mathrm{y}$ " $C$ " en función del lugar donde se recogió la información, y la asignación de un número correlativo que hace imposible la identificación de las jóvenes.

Recogida de datos: La recogida de datos se realizó mediante entrevistas semiestructuradas grabadas, con el propósito de describir la esencia de la experiencia humana vivida(11). En este sentido, las investigadoras ha creído acertada la recogida de los datos mediante una grabación para intentar recoger dicha experiencia en boca de las participantes y transcribirlas de forma íntegra. Se utiliza una entrevista semiestructurada con la idea de intentar centrar el tema objeto estudio con unas preguntas muy concretas y para facilitar la expresión y explicación de las respuestas. Las preguntas se elaboraron con anticipación y se plantearon a las personas participantes con una secuencia no prefijada, de carácter abierto, donde la entrevistada tuvo que construir las respuestas ${ }^{(17)}$. La elaboración previa del guión de la entrevista se hizo teniendo en cuenta los objetivos del estudio, básicamente se plantearon preguntas sobre la vivencia y la expresión de sentimientos y emociones asociados a la deformidad escoliótica del tipo: ¿Cómo te ves?, ¿Cómo crees que te ven los demás?, Explícame cómo influye la escoliosis en el desarrollo normal de tu vida... Durante la entrevista se utilizaron herramientas comunicativas como la escucha activa $o$ el silencio terapéutico, el objetivo fue que las personas manifestasen 
libremente sus sentimientos y percepciones respecto sus experiencias vividas con la escoliosis.

Este estudio forma parte de un estudio más amplio sobre las percepciones corporales y los aspectos emocionales de pacientes afectos de escoliosis idiopática adolescente. Dicho estudio forma parte a su vez, de una tesis doctoral inscrita en la Universidad de Alicante cuya defensa se realizó el 5 de febrero de 2016.

\section{RESULTADOS Y DISCUSIÓN}

La escoliosis se entiende como un problema más añadido a los ya existentes en esta etapa de constante cambio (adolescencia/juventud). Se trata de una época de la vida donde se producen cambios físicos y psíquicos de difícil asimilación, la deformidad supone un "problema" más del que preocuparse porque influye en los dos aspectos, el físico y el psíquico.

Cuando me lo detectaron y me dijeron lo del corsé estaba triste porque yo tengo otros problemas y se me van acumulando más. (C-2)

...antes de operarme me veía horrible...lo pasaba muy mal... a mí la escoliosis me ha traído muchos problemas...yo me he caído moralmente...por el dolor y estéticamente. (A-5)

\section{...tenía un problema y me afectaba mucho... (A-14)}

Las jóvenes describen su situación como "horrible" y la entienden como un problema de entre los muchos que se perciben con su edad puberal o madurativa. La adolescencia va acompañada de una percepción física y psicológica de cambios corporales que son percibidos como problemas para un individuo en constante cambio, y este nuevo problema en sus vidas, la escoliosis, se suma a todos los ya existentes $^{(18,20)}$. A todo esto se suman las dificultades propias de esta edad para expresar y/o manifestar sus sentimientos y emociones, existe una contención de emociones y sentimientos que van implícitas con este período de crecimiento.

... soy una chica que se guarda bastante los problemas para ella y se los traga y... bueno...el jueves en la UCl me dió un poco el bajón... y exploté... (Llora)... Siempre ha sido así...nunca he contado mis problemas ni nada... además, la escoliosis ha supuesto en general más problemas...más complejo... complejo y ya está. (A-10)

...prefiero no hablar de eso [se refiere a la escoliosis]...lo he pasado muy mal...no me siento cómoda... (A-3)

\section{(...) me preocupaba y me quedaba pensando... (A- 8)}

A ver a mí la escoliosis me ha traído muchos problemas... yo me he caído moralmente y...si pudiese apartar la palabra escoliosis mejor... pero tengo que convivir con ella toda mi vida...Me veía horrible...lo pasaba muy mal... (A-5)

La percepción corporal resulta el aspecto más subjetivo que las pacientes pueden compartir. Los sentimientos y la expresión verbal de cómo se ven a sí mismas señalan en algunos casos un déficit psicológico que debería tener soporte profesional en muchos casos. En la mayoría de los casos las jóvenes con escoliosis esperan que 
su cuerpo vaya cambiando con el tratamiento aplicado, desean una aceptación/integración en el grupo social al que pertenecen, necesitan sentirse aceptadas. Se desea formar parte de la normalidad física preestablecida socialmente para conseguir tener un problema menos de los muchos que refieren tener por su condición de jóvenes y adolescentes. También desean no tener que esconderse, no quieren esconder su cuerpo de una mirada ajena, de la mirada del grupo, ya que eso implica tener que aceptar un juicio social y tener que aceptar que se es diferente y que se tiene un defecto físico, aspecto éste asociado a un fracaso social y en consecuencia vital.

[Después de la cirugía]...espero que mi cuerpo vaya cambiando y ya está....ahora no tengo que esconderme y ya es un problema menos... (A-8)

No puedo evitar llorar porque me da mucha vergüenza... ¿por qué me pasa a mí y a los demás no? (A-7)

El compromiso estético ha sido considerado como un factor crítico para los pacientes con escoliosis idiopática. La angustia psicológica que experimentan estos pacientes a menudo se atribuye a la aparición de la deformidad del tronco. Las preguntas al respecto evidencian la idea de que estos pacientes sufren con regularidad los conflictos psicológicos, y cuando los conflictos se manifiestan la causa, normalmente, es el efecto cosmético ${ }^{(3,7,21)}$. En los estudios consultados se observa la angustia psicológica por la deformidad corporal a pesar de que ninguno de ellos da importancia a la presión social ejercida por una cultura que prioriza un canon estético y juvenil para alcanzar el éxito en la vida, la estética corporal se transforma en una prioridad y el deseo de pertenencia al grupo siendo un individuo "normal" forma parte de los anhelos de estas jóvenes deformes.

Uff... me costaba mucho porque me tenía que poner en bañador y que se me viera todo...siempre estaba tumbada... yo....al ponerme de pie siempre se me veía la chepa o de costado...se me notaba aquí los costados... la chepa... y quieras o no evitas el tumbarte porque se te nota...se pasa mal...muy mal. (A-5)

(...) la gente se ponía vestidos estrechos y yo no podía...iba a comprarme ropa con mis amigas y me sentía idiota porque me decían que la ropa me quedaba bien... pero yo me veía en el espejo y eso no era verdad... se veía toda la curva... [Llora]...llegué a ir sola a comprarme la ropa. (A-7)

La imagen de sí mismo es el dominio de la deformidad de columna vertebral más difícil de medir, especialmente porque las perspectivas de una persona pueden cambiar en las diferentes décadas de la vida, siendo la adolescencia y la juventud épocas especiales de cambios continuos que se suceden continuamente. Esto hace que sea un parámetro difícil de valorar a pesar de que la propia imagen es una de las características más importantes relacionadas con la deformidad de columna. La medición de la autoimagen es un proceso en constante evolución supeditado a la maduración de la persona y a su entorno sociocultural ${ }^{(22)}$. En general, todos los estudios revisados utilizan cuestionarios simples del tipo SRS-22, CAVIDRA, PHCSS... para ampliar el conocimiento de algunos factores que ponen de manifiesto la reducción de la calidad de vida de los pacientes afectados de escoliosis idiopática y que están específicamente relacionados con la depresión, el dolor de espalda, la capacidad física limitada y una negativa autopercepción corporal ${ }^{(7)}$. 
No aceptar la propia imagen corporal y observar cómo la anomalía se manifiesta y sale fuera de lo que se considera normal o aceptado por el grupo pone de manifiesto sentimientos y estados de afectación psicológica determinados. Cuando se pide a las pacientes que asocien la escoliosis a un sentimiento se les está pidiendo que recuerden su deformidad corporal y expliquen cómo se sienten, es entonces cuando aparecen palabras como vergüenza, tristeza, preocupación, miedo, amargura, complejo y rabia. Es este un momento especialmente difícil para ellas, porque han de escucharse a sí mismas y han de expresar con palabras algo que en algunos casos se oculta a los más próximos e incluso de forma inconsciente a uno mismo. Reconocer los sentimientos de vergüenza, tristeza, amargura y complejo es un esfuerzo psíquico que ocasiona llanto en algunas pacientes y que les hace reconocer su situación de deformidad corporal como una experiencia/vivencia horrible.

...sí, me daba vergüenza si se me notaba o si me decían algo...me ponía triste...me preocupaba y pensaba...y ahora qué, qué voy a hacer y que me harán y todo eso...estaba preocupada por todo... (A-8)

[La escoliosis]...a mí me aporta un poco de... como se llama...de vergüenza ponerme depende qué camisetas y eso...si más complejo... (A-10)

Tuve mucho miedo al principio, luego me quité el miedo porque si no, no me operaba. $(\mathbf{A}-5)$

Al principio cuando me lo detectaron pues a ver me dijeron que lo tenía que llevar durante un tiempo [se refiere al corsé]... y la verdad es que estaba un poco triste porque ya tengo otros problemas y... claro hay veces que se me van acumulando más y más... y ya tengo otros... (C-2)

En la línea de la imagen corporal, y coincidiendo con algunos estudios, las pacientes con escoliosis idiopática tienen una mayor propensión a desarrollar sentimientos de insatisfacción respecto a su apariencia corporal, en consecuencia, tienden a una falta de autoconfianza, a tener un sentido de inferioridad e incluso vergüenza. Se trata de una experiencia inquietante que obliga a los pacientes a enfrentarse al estrés, la negación, el miedo, la ira y la vergüenza ${ }^{(23,25)}$. Los sentimientos no son sólo experiencias mentales, suponen la alteración que el sujeto percibe en el resto de su organismo, esta alteración se manifiesta en forma de síntomas y estos a su vez, suponen un mecanismo de adaptación a una situación específica ${ }^{(26)}$.

Esta contención de sentimientos y sensaciones podría condicionar y facilitar una decisión quirúrgica donde no va a existir una valoración previa y profunda de los riesgos de la cirugía. Las jóvenes tienen el objetivo único de ser igual que los demás, de ser aceptadas por el grupo, de tener éxito en sus relaciones sociales y ese éxito lo proporciona una visión atractiva del cuerpo, visión que en su condición actual no tienen porque únicamente se ven deformes y su deseo más profundo es cumplir el compromiso social impuesto, un cuerpo joven y perfecto.

...tengo una cadera que está recta y la otra que esta... así, curva...vale... y eso a veces me da rabia...

(C-3)

Mi padre tiene la espalda curvada pero le da igual, a mi me da mucho apuro y no quiero que me vean...es horrible. [Llora] (A-7) 
La imagen del cuerpo constituye una parte muy importante de la autoestima de muchas personas, especialmente en la etapa de la adolescencia, donde el individuo se está formando y debe asumir un sinfín de cambios corporales que generan un déficit de autoconfianza, esta falta de autoconfianza puede llevar al pesimismo y a la ansiedad creciente, y el resultado es un deterioro del funcionamiento social y aislamiento psicológico integral, por tanto podría ser necesaria una rehabilitación física y social ${ }^{(23,25)}$. El efecto negativo de la deformidad de la columna en la percepción de la propia imagen y la apariencia parece ser el síntoma clínico predominante de la $\mathrm{EIA}^{(22)}$.

Las jóvenes que acceden a hablar sobre su problema sienten una profunda tristeza que manifiestan en forma de llanto cuando se les pide la expresión verbal de un sentimiento asociado a la escoliosis o cuando se les pide que expresen verbalmente las emociones que recuerdan con su experiencia. Recordar esa vivencia hace daño, duele, y refieren estados físicos y psíquicos concretos como pasarlo mal, verse horrible, caerse moralmente y sentirse muy hundida. Estos estados psíquicos podrían asociarse de forma genérica a la depresión o a estadios depresivos ocultos que podrían ocasionar y asociarse a una situación de estrés mantenida que desencadena una depresión reconocida verbalmente en algunos casos. Durante las entrevistas se observan algunos momentos en los que las pacientes son incapaces de articular palabras porque éstas se ahogan entre hipidos y llanto, expresan un sentimiento de tristeza muy profunda por la situación que padecen o han padecido, y son capaces de pedir perdón por la expresión incontrolable de esa tristeza, piden perdón por no poder controlar esas emociones que están surgiendo en un momento doloroso donde se recuerdan a sí mismas que son deformes y que no quieren ser así, estos momentos se escapan a su control consciente y fluyen de forma natural durante la entrevista. Son palabras imposibles de pronunciar y cuyo recuerdo provoca dolor, un dolor profundo que provoca hipidos (expresión de un momento ansioso), tristeza y llanto.

(...) Cuando estaba muy hundida hablaba con mis amigas o con mis padres... (A-8)

(...) la gente me ha animado mucho pero hay días que dices uff...no sé si podré... he estado deprimida [Llora]... (A-6)

\section{(...) yo me ha caído moralmente y lo he pasado muy mal en el sentido estético...}

si...no me veía nada bien ... antes de operarme me veía horrible...lo pasaba muy mal [Llora]... (A-5)

Tenía un problema y me afectaba mucho...yo no salía a la calle...me vengo a bajo con el tema de la escoliosis... [Respiración profunda] (A-14)

No puedo evitar llorar porque me da mucha vergüenza... [Llora, hipidos]... ¿por qué me pasa a mí y a los demás no?... (A-7)

Cuando una situación personal como la deformidad se vive como una pérdida de expectativas futuras se puede llegar a negar la existencia de aquello que produce dolor como mecanismo de defensa. La manera de evitar ese dolor psíquico es negar la evidencia, engañar al consciente estableciendo una ley del silencio que envuelve todo lo relativo a lo deforme, todo lo relativo a la escoliosis. Algunas pacientes viven un proceso de duelo por su escoliosis y se niegan a hablar de ella, en estos casos la pérdida que se experimenta es la pérdida de una imagen corporal socialmente aceptada según los cánones de belleza preestablecidos, y el no hablar de ello supone 
omitir la visión/expresión de su cuerpo, negándose a sí mismas unas expectativas futuras predeterminadas socialmente. Con este silencio consiguen no sentir dolor por la pérdida de lo deseado, intentan aparentar lo que ellas consideran "normalidad", aunque realmente saben que eso no es así.

En algunos casos esto se expresa inconscientemente cuando las adolescentes refieren no querer hablar del tema, se sienten incómodas cuando se les pide que expliquen su experiencia y se niegan a expresar cualquier aspecto relacionado con su escoliosis o con su experiencia pasada. Es en estos momentos cuando se puede observar la similitud con la etapa de negación en el proceso del duelo, se niegan a hablar de su deformidad, de su problema. Cuando la deformidad supone una pérdida tan importante que dificulta la comunicación de los sentimientos con las personas más allegadas (familiares, amigos,...) se puede hablar de un proceso de duelo donde la etapa de negación supone al mismo tiempo una dificultad para la expresión de unos sentimientos determinados y la palabra "escoliosis" pone al individuo en una situación de alerta y tensión estresantes, reconociendo que no quiere hablar del tema y que ve en aquellos que le rodean nerviosismo cuando ellos mismos intentan expresarse en cualquier aspecto, provocando con todo ello una conspiración del silencio en el sentido de necesidad de autoprotección por la incapacidad de enfrentarse al sufrimiento que genera la deformidad. Este aspecto se confirma también desde la perspectiva de los pacientes paliativos y sus cuidadores, el pacto de silencio se genera por la creencia de que decir "la verdad" perjudica a los pacientes, causándoles más ansiedad de la que tienen. En el caso de la escoliosis genera ansiedad la incapacidad de enfrentarse a la deformidad. El mantenimiento del pacto de silencio influye en el afrontamiento de la pérdida y en la calidad de vida ${ }^{(27)}$.

(...) no me siento cómoda...es un tema del que no quiero hablar [se refiere a la escoliosis]...no me siento a gusto...prefiero hablar de otras cosas [se coge las manos]. (A-2)

(...) me encuentro más tranquila si no hablo del tema... me cuesta un poco hablar de esto...no he hablado con nadie de esto...no... [Quejido] [Llora] (A-10)

(...) no suelo hablarlo con mis padres porque se ponen nerviosos...no hablo con mi hermana ni con mis amigos. Por favor, no quiero hablar de esto. (A-1)

Durante todo este proceso de pérdida de expectativas existen algunas adolescentes que refieren, de forma espontánea el acompañamiento de una serie de personas que hacen la función de soporte emocional, estas personas proporcionan apoyo psíquico, a ellas se les explican vivencias, sentimientos, experiencias y emociones. Estas personas parecen ser los conductores de ese estrés doloroso que se manifiesta con el problema escoliótico. El soporte más importante que refieren las pacientes está en su entorno más inmediato, sus padres, y más concretamente hacen referencia al rol de la madre y las hermanas (entendidas como cuidadoras en nuestra sociedad actual) ${ }^{(28)}$, también se habla de los amigos, compañeros del colegio y profesores, que ejercen de controladores en el día a día en el caso de aquellas pacientes portadoras de corsé.

Cuando estaba muy...muy hundida pues hablaba con las amigas o con mis padres y me desfogaba un poco pero a pesar de ello... pues...notaba que... que no me entendían del todo... (A- 8) 
Bien...el jueves cuando bajó mi madre lo estuvimos hablando y me dijo que... que nada que no podía vivir así que si me pasaba algo tenía que decirlo... que ella estaba allí... (A-10)

Mi madre la primera, mi hermana y todos siempre han estado apoyándome y dándome ánimos...bueno... a mi madre no le explicaba tanto porque mi madre siempre se preocupaba más... pero ella me lo notaba... pero a mis amigos sí les expliqué. Luego tengo una amiga que también está operada y salió en un mes... (A-5)

... yo lo expliqué a mis amigos... (C-1)

Las profesoras me iban vigilando y mis compañeros también... hasta inventaron un juego y para ver si me iba poniendo el corsé... la verdad es que me ayudaban mucho porque me han estado vigilando todo el rato y procuraban que me lo pusiera... cuando no me lo ponía... pues entonces se lo decían a un profesor...pero han visto que... se ve que he entrado un poco en razón... y me lo he ido poniendo... la verdad es que me ayudan bastante y tienen bastante cuidado conmigo para no hacerme daño ni nada. (C-2)

Se observa, a partir de los discursos que se recogen en las entrevistas, que los mecanismos de defensa personales utilizados por estas adolescentes para superar esos estados de estrés emocional que ocasionan los procesos de pérdidas son los pensamientos positivos y la búsqueda de información para validar esos pensamientos. Los pensamientos positivos ayudan a la persona a mantener su lucha interna, su ánimo, a sobrevivir frente a unos sentimientos negativos que exponen su mente al riesgo de sufrir un colapso emocional que derive en una depresión, haciéndoles sentir diferentes al resto del grupo, malformadas, horribles, en definitiva, no aceptadas y excluidas de la sociedad por su defecto, considerándose fuera de los cánones de belleza establecidos.

La búsqueda de información ayuda a validar los pensamientos positivos y obliga a la persona a buscar un soporte emocional fuera de su entorno más próximo, las jóvenes buscan e investigan en otras jóvenes con el mismo tipo de deformidad corporal, en foros, bibliografías, asociaciones, etc., el objetivo es validar sus conocimientos y de esa forma soportar mejor o entender mejor qué está ocurriendo en su cuerpo y qué puede ocurrir en un futuro. Comparten con otras chicas de su edad sus experiencias y las posibles soluciones a sus problemas. Buscan información para dar solución a su problema, se interesan básicamente por los posibles tratamientos, preguntan para superar el miedo, miedo a lo desconocido, al futuro, al dolor después de la cirugía y miedo a que su deformidad se cronifique y se mantenga en el tiempo o empeore ocasionando una deformidad mayor de la que ya padecen.

Miré unas webs...y miré fotos y radiografías y cosas así...pero para informarme más y ya está. $(\mathbf{A}-\mathbf{8})$

No sé... me conciencié busqué... hablé con mucha gente también y poco a poco decidí qué iba a hacer. (A-5)

Estuve un año preguntando y leía todo pues te animaba un montón... y también pensando pues que me voy a operar y me voy a poner bien...esto se va a solucionar... no te vas a quedar así...te apoyas a ti misma también. O así lo he hecho yo... también la gente me ha ayudado un montón, me metí en esto de Escoforo... 
porque te dan muchos ánimos... y lees las experiencias de otras personas...y las experiencias claro...cada uno vive la suya... (A-6)

Tengo una amiga que tiene lo mismo que yo pero está en mi país y ella me habla por whatsapp y me dice que "qué se le va hacer"... pero bueno a ella se le arregló un poquito con el corsé...no le daba tanta vergüenza como a mí. Ella salía y todo...pero yo no. Bueno decía que "había que seguir la vida" pero bueno ahora que le dije que me había operado se ha puesto muy contenta. (A-14)

La adaptación a su identidad exige tomar conciencia de uno mismo y de los retos. Las estrategias de afrontamiento como la actitud positiva y la determinación firme de querer superar las dificultades, el apoyo familiar, social y profesional, les va a permitir alcanzar la "normalidad". Observando que esta normalidad estará marcada por la discapacidad ${ }^{(29)}$.

Estos pensamientos positivos expresados verbalmente señalan la idea errónea de que el simple hecho de ser joven no implica tener problemas con la cirugía y esta misma cirugía parece ser la solución a todos sus problemas estéticos y a la vez psicológicos. Resulta curiosa la omisión de todos los efectos adversos de la intervención quirúrgica, como por ejemplo la pérdida de la movilidad vertebral, el alto riesgo de lesión medular, o bien la cicatriz tras la cirugía, estos efectos adversos no se comentan de forma específica, las pacientes seleccionan y filtran inconscientemente la información recibida en el preoperatorio y se quedan con el aporte estético que proporcionará la rectificación que aportará la fusión vertebral que, en definitiva, es aquello que buscan y anhelan.

Pues pensaba que ya quedaba menos y que... al ser joven pues que no tendría ninguna complicación al curarme... o al hacer ejercicio, por eso...o sea pensaba... ya queda menos, ya queda menos y ya está...no me preocupaba tanto...(A-8)

...he visto videos de chicas operadas que hacen ballet...hacer ballet con escoliosis $y$ operadas...he buscado por internet bailar con escoliosis, no soy la única y sé que se puede perfectamente... si aquí hay gente que esto no lo hace más allá seguro que hay gente que sí...muchas chicas que están operadas y pueden perfectamente hacer ballet no todo son movimientos de la espalda en el ballet...además yo el corsé sólo lo llevé por la noche y mis padres vieron el caso de mi hermana que también está operada y no me han obligado a llevarlo...yo me opero ahora mismo por mi futuro y por la estética. (A-11)

La corrección de la deformidad es observada por los pacientes como una mejoría en puntuaciones del dolor e imagen de sí mismo ${ }^{(31)}$. La imagen de sí mismo es el dominio de la deformidad de columna vertebral más difícil de medir, especialmente porque las perspectivas de una persona pueden cambiar en las diferentes décadas de la vida. Esto hace que sea un parámetro difícil de valorar a pesar de que la propia imagen es una de las características más importantes relacionadas con la deformidad de la columna ${ }^{(30,32,33)}$.

\section{CONCLUSIÓN}

> La escoliosis se entiende como un problema más añadido a los ya existentes en una etapa de constante cambio como es la adolescencia y la juventud. 
> La expresión verbal de cómo se ven a sí mismas estas jóvenes señala un déficit psicológico, se relatan sentimientos de vergüenza, tristeza, preocupación, miedo amargura, complejo y rabia. Expresar y relatar estos sentimientos verbalmente supone un esfuerzo psíquico que obliga a reconocer y recordar su situación de deformidad corporal como una vivencia horrible. La verbalización de sentimientos y emociones puede asociarse a estadios depresivos en los que las pacientes verbalizan "pasarlo mal", "verse horribles", "caerse moralmente" y "sentirse hundida". Aspecto éste que requeriría de una atención psicológica más especializada.

$>$ Cuando la deformidad se vive como una pérdida se puede hablar de un proceso de duelo, donde la negación es la no expresión y dificulta la comunicación de los sentimientos con las personas más allegadas (familiares, amigos,...)

$>$ El soporte emocional habitual se encuentra en el entorno más inmediato, los padres, y más concretamente la madre y las hermanas.

Los mecanismos de superación de la situación vivida son los pensamientos positivos y la búsqueda de información para validar estos pensamientos.

\section{REFERENCIAS}

1. Lasa Zulueta A. Experiencias del cuerpo y construcción de la imagen corporal en la adolescencia: vivencias, obsesiones y estrategias. Rev Psicopatología y Salud Ment del Niño y del Adolesc. 2003;2:53-74.

2. Miralles Sangro MT. Y el cuerpo no siempre es perfecto. Temperamentvm [Internet]. 2008;(7). Available from: http://www.indexf.com/temperamentvm/tn7/t6624.php

3. Pineda S, Bago J, Gilperez C, Climent JM. Validity of the Walter Reed Visual Assessment Scale to measure subjective perception of spine deformity in patients with idiopathic scoliosis. Scoliosis. 2006;1(18).

4. Carreon LY, Sanders JO, Polly DW, Sucato DJ, Parent S, Roy-Beaudry M, et al. Spinal Appearance Questionnaire: Factor Analysis, Scoring, Reliability and Validity Testing. Spine. 2011;36(18):E1240-1244

5. Climent JM, Cholbi Llobell F, Rodríguez Ruiz C, Mulet Perry S, Mendéjar Gómez F, Pradas Silvestre J. La medida de la salud en la escoliosis. Rehabilitación. 2009;43(6):299-305.

6. González Viejo MA, Avellanet Viladomat M, Sánchez Raya J, Bagó Granell J, Núñez Pereiro S, Climent Barberá JM. Escoliosis y Cifosis. Estado de la situación. Euromedice. 2009. 189 p.

7. Tsutsui S, Pawelek J, Bastrom T, Lenke L, Lowe T, Betz R, et al. Dissecting the Effects of Spinal Fusion and Deformity Magnitude on Quality of Life in Patients With Adolescent Idiopathic Scoliosis. Spine. 2009;34(18):E653-658 .

8. Sanders JO, Carreon LY, Sucato DJ, Sturm PS, Diab M. Preoperative and Perioperative Factors Effect on Adolescent Idiopathic Scoliosis Surgical Outcomes. Spine. 2010;35(20):1867-1871.

9. Parent EC, Hill D, Mahood J, Moreau M, Raso J, Lou E. Discriminative and Predictive Validity of the Scoliosis Research Society-22 Questionnaire in Management and Curve-Severity Subgroups of Adolescents With Idiopathic Scoliosis. Spine. 2009;34(22):2450-2457.

10. D’Agata, E.; Rigo, M.; Pérez-Testor, C.; Casanovas, N.; Castellano-Tejedor C. Emotional indicators in young patients with Idiopatic Scoliosis: a study through the 
drawing of Human Figure. Scoliosis [Internet]. 2014;9:24. Available from: http://www.scoliosisjournal.com/content/9/1/24

11. Burns N, Grove SK. Investigación en enfermería. Desarrollo de la práctica enfermera basada en la evidencia. Barcelona: Elsevier España S.L.; 2012.

12. Martín-Crespo Blanco MC, Salamanca Castro AB. El muestreo en la investigación cualitativa. Nure Investig. [Internet] 2007;27. Available from: http://www.nureinvestigacion.es/OJS/index.php/nure/article/view/340

13. Polit D, Hungler BP. Investigación científica en ciencias de la salud. México: McGraw-Hill Interamericana; 2000. 725p.

14. González Morales A. Los paradigmas de investigación en las ciencias sociales. ISLAS. 2003;45(138):125-35.

15. Álvarez Garcia de Quesada L, Núñez Giralda A. Escoliosis idiopática. Rev Pediatr Aten Primaria. 2011;13:135-146.

16. De Castro Correa G. Psicología clínica. Fundamentos existenciales. $2^{\mathrm{a}}$ ed. Universidad del Norte. 2011. 23-45 p.

17. Vargas Jiménez I. La entrevista en la investigación cualitativa: nuevas tendencias y retos. Rev CAES. 2012;3(1):119-139.

18. Olesti Baiges M, Martín Vergara N, Rera Solé A, de la Fuente García M, Bofarull Bosch JM, Ricomá de Castellarnau G. Valoración de la propia imagen corporal en adolescentes femeninas de 12 a 21 años de la ciudad de Reus. Enfermería Clínica. 2007;17(2):78-84.

19. Rivarola MF. La imagen corporal en adolescentes mujeres: su valor predictivo en trastornos alimentarios. Fundamentos en Humanidades [Internet]. 2003;4(007008):149-61. Available from: http://www.redalyc.org/pdf/184/18400808.pdf

20. Sontag S. La enfermedad y sus metáforas. Capellades, Barcelona; 1989.

21. Weiss HR, Werkmann M, Stephan C. Brace related stress in scoliosis patientscomparison of different concepts of bracing. Scoliosis [Internet]. 2007;2(10). Available from: http://www.scoliosisjournal.com/content/2/1/10

22. Bonilla Carrasco M. Imagen percibida en escoliosis idiopática adolescente: Revisión integrativa de la literatura. Rev Esc Enferm USP [Internet]. 2014;48(4):74858. Available from: http://www.scielo.br/pdf/reeusp/v48n4/es_0080-6234-reeusp-4804-748.pdf

23. G Aulisa A, Guzzanti V, Perisano C, Marzetti E, Specchia A, Galli M, et al. Determination of quality of life in adolescents with idiopathic scoliosis subjected to conservative treatment. Scoliosis [Internet]. 2010;5(21). Available from: http://www.scoliosisjournal.com/content/5/1/21

24. Sapountzi-Krepia D, Psychogiou M, Peterson D, Zafiri V, lordanopoulou E, Michailidou $F$, et al. The experience ob brace treatment in children/adolescents with scoliosis. Scoliosis. 2006;1(8).

25. Zhang J, Wang D, Chen Z, Gao J, Yu X, Sun H, et al. Decrease of self-concept in adolescent patients with mild to moderate scoliosis after conservative treatment running head: Self-concept in adolescent scoliosis patients. Spine 2011;36(15):E1004-1008.

26. Castilla del Pino C. Teoría de los sentimientos. Ensayo. Barcelona: Tusquets. 2000. $377 \mathrm{p}$.

27. Cejudo A, López B, Duarte M, Crespo MP, Coronado C, de la Fuente C. El pacto de silencio desde la perspectiva de las personas cuidadoras de pacientes paliativos. Enferm Clin.2015; 25(3): 124-132

28. Siles J, Solano C. Estructuras sociales, división sexual del trabajo y enfoques metodológicos. La estructura familiar y la función socio-sanitaria de la mujer. Investigación y Educ en Enfermeria/Medellín. 2007;25(1):66-73 
29. Font I, Llauradó M, Pallarés A, García F. Factores psicosociales implicados en la amputación. Revisión sistemática de la literatura. Aten Primaria [Internet].2015. Available from: http://dx.doi.org/10.1016/j.aprim.2015.04.009

30. Carreon LY, Sanders JO, Diab M, Sucato DJ, Sturm PF, Glassman SD. The minimun clinically important difference in Scoliosis Research Society-22 Appearance, Activity, and Pain Domains after surgical correction of adolescent idiopathic scoliosis. Spine. 2010;35(23):2079-2083.

31. Sue-Min Lai SM, Burton DC, Asher MA, Carlson BB. Converting the SRS-24, SRS-23, y SRS-22 to the SRS-22r: Establishing conversion equations using regression modelling. Spine. 2011; Febrero 1.

32. Tsutomu A, Shohei M, Toshiaki K, Tetsuharu N, Takana K, Kazuhisa T. Longterm clinical outcomes of surgery for adolescent idiopathic scoliosis 21 to 41 years later. Spine. 2012;37(5):402-405.

33. Vecina Dormido R, Kesting Jiménez ASM, Martínez-Fuentes J, Moya-Faz FJ. Escoliosis idiopática y autoconcepto en el adolescente. RevFisioter (Guadalupe). 2009;8(2):27-36.

Recibido: 19 de noviembre 2015; Aceptado: 24 de febrero 2016

ISSN 1695-6141

() COPYRIGHT Servicio de Publicaciones - Universidad de Murcia 